\title{
The Concept of Existence in Mathematics*
}

\author{
El concepto de existencia en matemáticas
}

Pablo M. Jacovkis ${ }^{\dagger}$

\begin{abstract}
Resumen
Afirmamos que, desde un punto de vista pragmático, los matemáticos tratan los objetos matemáticos como si fueran reales. Si una teoría es consistente, los teoremas se descubren (a veces con análisis no necesariamente diferentes de los aplicados en ciencias naturales) y las demostraciones se inventan; la tecnología moderna no puede existir sin aceptar la ley del tercero excluido; una demostración constructiva puede suministrar nuevas ideas o métodos pero, desde el punto de vista matemático, una demostración no constructiva es tan sólida como una constructiva. En consecuencia, ningún matemático, puro o aplicado, prescinde del axioma de elección; por otra parte, aunque según se acepte o no la hipótesis del continuo pueden aparecer distintos teoremas y objetos, no existe -al menos hasta ahora- ningún teorema importante aplicable al mundo real que dependa de aceptar o no dicha hipótesis. Los objetos matemáticos construidos por matemáticos aplicados son a menudo tan útiles como los objetos físicos, incluso aquellos objetos que fueron creados mediante métodos computacionales o probabilísticos.
\end{abstract}

Palabras clave: existencia matemática - descubrimiento matemático - invención matemática

\begin{abstract}
We assert that, from a pragmatic point of view, mathematicians treat mathematical objects as if they were real. If a theory is consistent, theorems are discovered (sometimes with analyses not necessarily different from those applied in sciences) and proofs are invented; modern technology cannot exist without accepting the law of excluded middle; a constructive proof may provide new ideas or methods but, from a mathematical point of view, a non-constructive proof is as sound as a constructive one. Accordingly, no mathematician, pure or applied, gets by without the axiom of choice; on the other hand, although different theorems and objects may appear depending on the acceptance or not of the continuum hypothesis, no important theorem applicable to the real world exists - at least until now - which depends on accepting or not this hypothesis. Mathematical objects built by applied mathematicians are often as useful as physical objects, even those objects created via computer-assisted or probabilistic methods.
\end{abstract}

Keywords: mathematical existence - mathematical discovery - mathematical invention

\footnotetext{
${ }^{*}$ Recibido: 16 de Febrero 2016. Aceptado con revisiones: 19 Septiembre 2016.

† Universidad Nacional de Tres de Febrero y Universidad de Buenos Aires, Argentina. Para contactar al autor, por favor escribir a: pjacovkis@untref.edu.ar.

Metatheoria 7(2)(2017): 17-23. ISSN 1853-2322.

(C) Editorial de la Universidad Nacional de Tres de Febrero. Publicado en la República Argentina.
} 


\section{Introduction}

This work is focused from a pragmatic point of view, that is, we shall try to show and to exemplify how an applied mathematician - or a computer scientist, a physicist, an engineer or any specialist who uses mathematics as a tool of his or her work - consciously or unconsciously considers the mathematical objects as real entities that "exist", provided with concrete properties; in that sense, the concept of existence is very similar to the notion of existence defined by Mario Bunge, for instance in Bunge (1985). Anyway, there is a caveat; as Bunge very clearly explains in Bunge (1997), he discusses pure mathematics, from the viewpoint of moderate mathematical fictionism; some of his assertions are very clear in that sense: "fictionism is [...] quite true of pure mathematics" (p. 51), "pure mathematics is not about the real world or about experience" (p. 51), "pure mathematics is ontologically neutral and, more precisely, a great (though not arbitrary) fiction" (p. 57), "[p]ure mathematics, then, is not about concrete or material things such as photons or societies. It is about conceptual or ideal objects" (p. 59).

By the way, this was the idea that Cantor had of mathematical objects when he created the set theory. In the first paragraph of his seminal work, Cantor (1895) says that "by an 'aggregate' we are to understand any collection [...] of definite and separate objects of our intuition or our thought" (unsrer Anschauung oder unseres Denkens). Therefore, as well as we define concrete and abstract nouns, we may define real and conceptual objects. Furthermore, for most pure mathematicians, the situation is the same: for the daily work in mathematics, pure or applied, the difference between real and conceptual objects is vague, practically inexistent.

In mathematics - as in the other sciences - two powerful forces move research and researchers: on the one hand, curiosity and thirst for knowledge - a feeling that in a sense could be compared to the famous answer of the great mountaineer George Mallory to the question "Why climb Mount Everest?": "Because it's there"; on the other hand the wish to apply the results obtained. We adopt the point of view of the applied mathematicians, that is, the mathematicians inspired by this second force. In the end, mathematics is an incredibly powerful tool to solve concrete problems and - except for issues with philosophical or epistemological interest, or studies in foundations of mathematics, or for theories introducing new potentially enriching approaches - in the long run the filter which separates lively from dying theories is their usefulness. Even the theory of numbers, originally studied only because of its fascination, is now (to the despair of G. H. Hardy, if he were alive) crucial in cryptography. And among the branches of mathematics currently studied without a potentially clear application, it remains to be seen which of them will survive in the future. Kolmogorov's theory of probability is stunningly beautiful but, had it not been so useful, it would have been abandoned long ago.

\section{Discovery and invention}

Let us assume that all the theories which we discuss are consistent, including those whose consistency has not been proved. For, if a theory is inconsistent, and leads to contradictions, no mathematical object exists in it. And we assume, besides, that the law of excluded middle holds, and our approach is not constructivist, in the sense that knowing that a mathematical object exists does not mean that we can show or locate it. Given that all modern technology is influenced by computer science, it is worth remembering that the conjunction between logic based in the law of excluded middle and electrical engineering permitted the creation of modern electronic computers: the outstanding Shannon (1940) dissertation where he established the correspondence between propositional logic and electric circuits is clear: electrons flow in an electric circuit depending on the truthfulness of falsehood of a proposition. (This does not mean that one should abandon other logics, as one does not abandon nonEuclidean geometries: in other contexts they may be useful.)

As a first approximation, then, when a (consistent) mathematical theory is formulated, all the theorems deducible from it are automatically established. The task of the mathematician consists in "discovering" these theorems, not in inventing them. A shorter, more elegant, proof may be invented; but the theorem "exists" independently of the proof. Anyway, this division is perhaps too categorical: 
Gowers (2011), when analysing whether mathematics is discovered or invented - and, more generally, when analysing the concept of discovery and the concept of invention -, defines a more diffuse boundary between what is discovered and what is invented in mathematics: essentially, he says, the feature that distinguishes what is invented from what is discovered is "the control that we have over what is produced".

Of course, no mathematician formulates a new theory "to see what happens". When a new theory is created, its creators have in mind what the theory is useful for, or that the theory is necessary to demonstrate some results whose intuitive idea they already have conceived, and formalization is necessary. And along the process of proving theorems new mathematical objects are being created, which (conceptually) exist. As a matter of fact, those objects, although without material existence, are not comparable to the imaginary objects (or characters) created by, say, a writer: they are subject to very strict conditions (some of them perhaps unknown to us). When the Banach spaces were "created", they could not have arbitrary characteristics. They had to behave in a certain way; we could not, with a stroke of imagination, force them to have properties they cannot have; in fact, outstanding mathematicians have investigated those spaces and "discovered" a lot of fascinating properties. Du Sautoy (2011) tells that one of his "proudest moments as a mathematician was constructing a new symmetrical object whose subgroup structure is related to counting the number of solutions modulo $p$ of an elliptic curve". Although du Sautoy says that he did not build it "physically", it is clear that the object lives "in the abstract world of mathematics" (that is what du Sautoy says): for all practical purposes (and du Sautoy is a pure mathematician), inside mathematics the object exists.

\section{Constructive and non-constructive proofs}

The proof of a theorem may be "constructive", like the proof of the theorem of existence and unicity of an ordinary differential equation under reasonable hypotheses, or may be non-constructive (for instance, a proof by reductio ad absurdum), like the traditional proof that the square root of two is irrational. Does the concept of existence depend on the type of demonstration used? According to the criterion followed in this work, it does not.

Why? During many centuries any proof of the irrationality of the square root of two was by reductio ad absurdum. Nevertheless, that non-constructive proof allows us to claim that the set of irrational numbers is non-void, and so to affirm (non-constructively) that this set exists. Interestingly, nobody can work efficiently in mathematics without accepting the existence of the real numbers (the rational plus the irrational) as a non-countable set, although only a countable subset of it may be exhibited (the rational, the algebraic, and some transcendental numbers). Of course a constructive proof is often better in the sense that it is more directly useful to have an object "of flesh and blood" (for instance, the constructive proof of the theory of existence and unicity of a solution of an ordinary differential equation suggests the explicit Euler method for its numerical solution), but that does not mean that something obtained by means of a non-constructive proof exists "less" than something obtained by means of a constructive proof. By the way, objects created with non-constructive proofs existed often before the corresponding constructive proofs, and sometimes no constructive proof of a theorem has yet been found; for instance, no constructive proof exists of the fact that $\left(l^{\infty}\right)^{*}$ contains properly $l^{1}$.

Besides, non-constructive proofs may be useful "in a constructive way" for other reasons: suppose that by means of a non-constructive proof the existence and unicity of the solution of a general problem are guaranteed, and suppose that in a particular case a numerical solution approximates a solution: we know that that solution is the solution searched, because we know (thanks to the nonconstructive proof) that the solution is unique.

With this approach, the situation is not particularly different to the situation of the professor who, in front of his or her students, asserts that one of them has more hairs than the others; obviously, no one can know who this student is. Or to the situation of the observer of a demonstration in which 
500,000 citizens have participated, who says that there exists one demonstrator who has more hairs than the others. Although for the mathematical object built with a non-constructive proof the impossibility of exhibiting it is absolute, and for the examples with hair the impossibility is technical, in fact the boundary between both is diffuse: it does not seem likely (at least in a not too distant future) that we find the way (and the necessity) of computing exactly the number of hairs of a crowd of $500,000 \ldots$

Besides, from our pragmatic point-of-view, for instance Maheara (1984) invented an extremely clear and elegant non-constructive proof of the Jordan curve theorem. Assuming that no constructive proofs could be found of this theorem, probably no mathematician would be willing to get by without this theorem. And, more generally, mathematics would be much poorer without using the axiom of choice, which is non-constructive.

\section{Completeness and independence}

Now, from Gödel's incompleteness theorem we know that - in the underlying theory - there are propositions $p$ that are true (or their negations $\sim p$ are true) but that cannot be proved with the tools of the theory. For instance, if Andrew Wiles had not proved Fermat's last theorem, it is possible that some mathematicians would have begun to think that perhaps that theorem were a concrete example of Gödel's theorem: that the theorem were true (or false) but the theory needed to be "reinforced" to be able to find a proof. This situation happens with many conjectures. For instance, with the Goldbach's conjecture: every integer greater than two can be expressed as the sum of two primes. We are sure that the conjecture is true or false, but perhaps we cannot prove its truthfulness or falsehood inside the theory.

Other situation is also possible: that a proposition is independent of the rest of the theory, and the proposition (or some variant of its negation) may be incorporated to the theory as a new axiom, so that the broadened theory continues being consistent. That is what happened, of course, with geometry and Euclid's fifth postulate: if we do not accept it, we have a non-Euclidean geometry instead of the traditional Euclidean one. And that is what happens with the axiom of choice and with the continuum hypothesis.

Regarding the axiom of choice, there are no practical problems: except the constructivists, all mathematicians accept it, because the mathematics obtained including it is so much richer than the mathematics obtained without accepting it, that it is almost impossible to resist the temptation of using it, that is, of creating mathematical objects that exist only thanks to the axiom of choice or some of its equivalent propositions, such as Zorn's lemma. With the Zermelo-Fraenkel set theory plus the axiom of choice (the ZFC set theory) the mathematical theories built are not only extraordinarily beautiful and rich, but also extraordinarily useful and applicable. Elegance and pragmatism go hand in hand.

But with the continuum hypothesis $(\mathrm{CH})$, the situation is more ambiguous. Since Paul Cohen showed that $\mathrm{CH}$ is independent of the ZFC theory, we may accept $\mathrm{CH}$, and then we have a theory, with its theorems and its objects, or may not accept it, having then new theories with their theorems and their new existing objects (among them, of course, a set whose cardinality is greater than $\boldsymbol{\aleph}_{0}$ and less than c). But, until now, no important theorems applicable to the real world exist which depend on accepting or not $\mathrm{CH}$. Anyway, be they useful or not, be they important or not, different theorems exist accepting or not $\mathrm{CH}$, and the mathematical objects created exist (conceptually). This commentary, naturally, does not underestimate the enormous importance of the studies and analysis of $\mathrm{CH}$ in the foundations of mathematics. Simply, until now, real life mathematical models do not take into account whether this hypothesis holds or does not hold, but nothing forbids that, in the future, important and useful models may appear based in $\mathrm{CH}$ or in some variant of its negation.

We may claim that this discussion is posed for objects whose cardinality is infinity, due to the fact that all is (or seems) simpler with finite objects. But, on the one hand, the sets of natural and real 
numbers are infinite objects which we accept unreservedly; and, on the other hand, the Fischer-Griess monster, the finite group $M$ with

$$
\begin{aligned}
& 2^{46} \cdot 3^{20} \cdot 5^{9} \cdot 7^{6} \cdot 11^{2} \cdot 13^{3} \cdot 17 \cdot 19 \cdot 23 \cdot 29 \cdot 31 \cdot 41 \cdot 47 \cdot 59 \cdot 71 \\
& =808017424794512875886459904961710757005754368000000000 \\
& =808,017,424,794,512,875,886,459,904,961,710,757,005,754,368.000,000,000 \\
& \approx 8 \times 10^{53}
\end{aligned}
$$

elements, is probably much more difficult to visualise mentally, except for some specialist in finite groups, than the real numbers.

It is interesting to comment, with regard to the Fischer-Griess monster, that Borcherds (2002) says that "[t]he monster was originally predicted to exist by B. Fischer and by R. L. Griess in the early 1970s. Griess constructed it a few years later in an extraordinary tour de force" and Wikipedia (https://en.wikipedia.org/wiki/Monster_group) remarks that Fischer, Conway, Norton and Thompson discovered other groups as subquotients. Wikipedia employs the verb "discover": the groups were "there", it was necessary simply to find them; the construction by Griess seems more a discovery than an invention. And regarding the prediction of its existence, it would be worth analyzing in detail whether there is any difference between the reasoning leading to its prediction, and then to its exhibition, and the reasoning leading to the prediction, and then to the localization, of the planet Neptune.

\section{Existence in applied mathematics}

Focusing now our interest specifically in applied mathematics, we may mention the Lax equivalence theorem: for a consistent finite difference method for solving a well-posed linear initial value problem of partial differential equations, the method is convergent if and only if it is stable (Lax and Richtmyer, 1956). Lax's proof has a non-constructive part. No applied mathematician stopped using (and using successfully) the theorem due to the non-constructive part.

Furthermore, one of the most attractive areas of applied mathematics is, in the field of numerical solution of partial differential equations, the finite element theory, due to its elegant theoretical basis as well as to its powerful applications. And, according to the type of numerical problem to solve, different finite elements are "invented": the Argyris triangular elements, the Lagrange elements, the Hermite elements, the Morley elements; quadrangular finite elements, and so on. Each finite element invented, or constructed, is a mathematical object, and so it is real (conceptually); when one runs on a computer a (correct) program including the finite element (correctly) chosen, the program gives back exactly what it should, with exactly the accuracy required.

\section{Experimental mathematics}

In their 1953 seminal work (Fermi et al., 1955), Fermi, Pasta, Ulam and Tsingou performed numerical simulations with a system of near-neighbour coupled equations, which included weak nonlinear quadratic or cubic terms, simulating vibrating strings. They observed that, contrary to the thermalization that intuition suggested, the computer results showed a very complicated quasi-periodic behaviour. Some years later, this strange phenomenon could be related to the soliton theory and the Korteweg-de Vries equation (Zabusky \& Kruskal, 1965). That is, it was possible to associate the existence of a mathematical object (the already known Korteweg-de Vries equation) to a physical phenomenon observed for the first time by the computer ("in silico"). Similarly, many years later, the Feigenbaum constant that appears in chaos theory was detected, as if it were a physical object, before its existence could be mathematically proved. In fact, Feigenbaum (1979) found this constant in 1976 and Landford (1982) invented a computer-assisted proof to show that it was indeed a constant. And it is not yet known whether Feigenbaum constant is a transcendental number or not. But it is clear that 
either it is transcendental or not; somebody will "discover" that the constant is transcendental, or that it is not transcendental.

\section{7. "Probability" of existence}

An additional discussion deserves the following case: Glimm (1965) proved that, under certain conditions, for a system of hyperbolic conservation laws it is possible to construct, by means of a probabilistic algorithm, a succession of approximate solutions which converge, with probability one, to the only theoretical solution of the system. That is, the solution of the system exists, and is unique, with probability one. What assurance we have then of the actual existence (in the conceptual sense) of this solution? In other words, when we are working with a particular system of hyperbolic conservation laws, and we want to be sure that the solution exists and is unique, how do we know that we have not had bad luck and the system belongs to the set (of probability zero) without solution? In practice, this alternative does not happen: using Glimm's theorem Chorin (1976) designed a probabilistic numerical method (the random choice method) to solve nonlinear hyperbolic systems of conservation laws, and the method works and is applied.

\section{Conclusion}

Summing up: focusing the discussion about the existence of mathematical objects from a pragmatic point of view, point of view which is adopted by any mathematician who works in problems of physical (or economic, or social) reality, mathematical objects may be treated as very concrete objects, whose existence and properties are guaranteed. In many senses objects not totally known may be studied as if the mathematician were an explorer. Most pure mathematicians, and all applied mathematicians, are Platonic, like it or not.

\section{References}

Borcherds, R.E. (2002), "What is the Monster?", Notices of the American Mathematical Society 49(9): 1076-1077.

Bunge, M. (1985), Treatise on Basic Philosophy, Vol. 7: Epistemology and Methodology III: Philosophy of Science and Technology Part I, Dordrecht: Reidel.

Bunge, M. (1997), "Moderate Mathematical Fictionism”, in Agazzi, E. and G. Darvas (eds.), Philosophy of Mathematics Today, Dordrecht: Kluwer, pp. 51-71.

Cantor, G. (1895), "Beiträge zur Begründung der transfiniten Mengenlehre I”, Mathematische Annalen 46(4): 481-512. English translation: "Contributions to the Founding of the Theory of Transfinite Numbers Article I (1895)", in Cantor, G. (1915), Contributions to the Founding of the Theory of Transfinite Numbers, New York: Dover, pp. 85-136.

Chorin, A.J. (1976), "Random Choice Solution of Hyperbolic Systems", Journal of Computational Physics 22(4): 517523.

Du Sautoy, M. (2011), "Exploring the Mathematical Library of Babel”, in Polkinhorne, J. (ed.), Meaning in Mathematics, Oxford: Oxford University Press, pp. 17-25.

Feigenbaum, M.J. (1979), “The Universal Metric Properties of Nonlinear Transformations”, Journal of Statistical Physics 21(6): 669-706.

Fermi, E., Pasta, J. and S. Ulam (1955), Studies of Nonlinear Problems, Los Alamos Document LA-1940, Los Alamos: Los Alamos Scientific Laboratory.

Glimm, J. (1965), "Solutions in the Large for Nonlinear Hyperbolic Systems of Equations", Communications on Pure and Applied Mathematics 18(4): 697-715.

Gowers, T. (2011), “Is Mathematics Discovered or Invented?", in Polkinhorne, J. (ed.), Meaning in Mathematics, 
Oxford: Oxford University Press, pp. 3-12.

Landford III, O.E. (1982), "A Computer-assisted Proof of the Feigenbaum Conjectures", Bulletin of the American Mathematical Society 6(3): 427-434.

Lax, P.D. and R.D. Richtmyer (1956), "Survey of the Stability of Linear Finite Difference Equations", Communications on Pure and Applied Mathematics 9(2): 267-293.

Maheara, R. (1984), “The Jordan Curve Theorem via the Brower Fixed Point Theorem”, American Mathematical Monthly 91(10): 641-643.

Shannon, C. (1940), A Symbolic Analysis of Relay and Switching Circuits, M.S. Dissertation, Cambridge, MA: Massachusetts Institute of Technology.

Zabusky, N.J. and M.D. Kruskal (1965), "Interactions of Solitons in a Collisionless Plasma and the Recurrence of Initial States", Physical Review Letters 15(6): 240-243. 